\title{
Review of: "Long term prognosis in patients with pulmonary hypertension undergoing catheter ablation for supraventricular tachycardia"
}

\author{
SHUENN-NAN CHIU
}

Potential competing interests: The author(s) declared that no potential competing interests exist.

The author analyzed the ablation outcome in $23 \mathrm{PH}$ patients with 30 SVT. The procedure success rate was 83\%, high in AF and AVNRT and low in AT. Antiarrhythmic drugs, serum brain natriuretic peptide levels and number of hospitalizations per year significantly decreased after RFCA. Five and ten-years Kaplan-Meier free survival rate was $74 \%$ and $43 \%$ respectively. The presence of SVT after ablation was associated with poor long term outcome. Although an well written report, there were several major drawback exists.

Major

1. Many of the patients were CHD patients, and it is not surprised to see AF and IART as most common arrhythmia in these patients. The number of cases of PAH without CHD is less than 10. For patients of CHD with SVT, the benefit of catheter ablation on the heart failure symptoms, reduction of AAD use, and decrease hospitalization were already known. This article added little new. The author should describe separately about PAH without CHD to see the benefit of catheter ablation.

2. The author found those with presence of SVT after ablation had poorer long-term outcome. However, many of the arrhythmia belonged to AT, which may be the result of PAH disease progression. What the author suggested "to maintain a stable sinus rhythm after RFCA as an important treatment goal" may be difficult to achieve.

What is the pre-ablation risk factors of later occurrence of SVT and even mortality may be more important.

3. As a retrospective study, the author can't know actually the indication of catheter ablation in these patients. What is the cut-point of PAP/PVR at which the author would consider the risk is too high to ablate? This is what the readers want to know.

4. The author should describe the cause of mortality. Did they associate with the SVT?

Minor

1. Why there is no patients of atrial arrhythmia from left atrium in current study. Is it caused by selection bias because of high risk of transseptal puncture for left side arrhythmia? Or is it true that all atrial arrhythmia for PAH originate from right atrium?

2. Acute success or failure were defined as termination of an SVT circuit during application of radiofrequency energy, or no subsequent inducibility by programmed stimulation. "Or" should be 
revised as "and".

3. In figure 2 and 3, the legends of figure doesn't match the figures. 